\title{
PERFIL EPIDEMIOLÓGICO DA HEPATITE C NO BRASIL NO PERÍODO 2013 - 2018
}

\author{
EPIDEMIOLOGICAL PROFILE OF HEPATITIS C IN BRAZIL IN THE \\ PERIOD 2013-2018
}

\author{
Thamara Rodrigues de Melo ${ }^{1}$ \\ Giulian César da Silva Sá ${ }^{2}$ \\ Laísa Vilar Cordeiro ${ }^{3}$ \\ Pedro Thiago Ramalho de Figueiredo ${ }^{4}$ \\ Sávio Benvindo Ferreira ${ }^{5}$ \\ Aleson Pereira de Sousa
}

\begin{abstract}
RESUMO: A hepatite $C$ é uma das principais causas de doença hepática crônica em todo o mundo. A estimativa indica que o Brasil é um país com prevalência intermediária, variando entre $1 \%$ e $2 \%$. Diante desse contexto, o objetivo deste estudo relatar perfil epidemiológico da hepatite $C$ no Brasil com enfoque nos aspectos relacionados na distribuição da infecção por gênero, faixa etária, etnia, região do país e fontes de transmissão. Trata-se de um estudo documental, descritivo, de coleta retrospectiva, por meio de casos notificados pelo DATASUS referentes aos agravos de hepatite $C$ diagnosticados no Brasil entre 2013 a 2018. Nesse estudo, a maior prevalência da faixa etária de 40 a 59 anos $(53,4 \%)$, do sexo masculino (57\%), da raça branca $(53,6 \%)$. No entanto, a escolaridade e fonte de transmissão foram notificados maioria como "ignorado". Pela ausência de vacina ou profilaxia pósexposição eficaz, o foco principal da prevenção está na investigação epidemiológica, como uma ferramenta imprescindível para reconhecimento e o entendimento da dinâmica para controle da epidemia por HCV.
\end{abstract}

Palavras chave: Brasil. Doenças transmissíveis. Epidemiologia. Hepatite C.

\footnotetext{
${ }^{1}$ Doutoranda do Programa de Pós-graduação em Produtos Naturais e Sintéticos Bioativos (PgPNSB)/ UFPB (Área de concentração: Farmacologia).

${ }^{2}$ Doutorando do Programa de Pós-graduação em Bioquímica PPGBIOQ/ UFRN (Área de concentração: Bioquímica).

${ }^{3}$ Doutoranda do Programa de Pós-graduação em Produtos Naturais e Sintéticos Bioativos (PgPNSB)/ UFPB (Área de concentração: Farmacologia).

${ }^{4}$ Doutorando do Programa de Pós-graduação em Produtos Naturais e Sintéticos Bioativos (PgPNSB)/ UFPB (Área de concentração: Farmacoquímica).

${ }^{5}$ Professor Doutor da Unidade Acadêmica de Ciências da Vida da Universidade Federal de Campina Grande, Centro de Formação de Professores.
} 
ABSTRACT: Hepatitis $C$ is one of the leading causes of chronic liver disease worldwide. The estimate indicates that Brazil is a country with intermediate prevalence, ranging from $1 \%$ to $2 \%$. In this context, the aim of this study to report an epidemiological profile of hepatitis $C$ in Brazil focusing on aspects related to the distribution of gender infection, age group, ethnicity, region of the country and transmission sources. This is a documentary, descriptive, retrospective collection study, through cases reported by DATASUS related to hepatitis $C$ injuries diagnosed in Brazil between 2013 and 2018. In this study, the highest prevalence of the age group from 40 to 59 years (53.4\%), male (57\%), white (53.6\%). However, schooling and transmission source were notified most as "ignored". Due to the absence of vaccine or effective post-exposure prophylaxis, the main focus of prevention is on epidemiological investigation, as an indispensable tool for recognition and understanding of the dynamics for control of the epidemic by HCV.

Keywords: Brazil. Communicable diseases. Epidemiology. Hepatitis C. 


\section{INTRODUÇÃO}

A infecção pelo vírus da hepatite $C(\mathrm{HCV})$ é uma das principais causas de doença hepática crônica no mundo e constitui um importante problema de saúde pública. A hepatite $\mathrm{C}$ é a principal causa de transplante hepático em países desenvolvidos, e estimativas indicam que complicações decorrentes da infecção crônica pelo HCV, como cirrose descompensada e/ou carcinoma hepatocelular, devem aumentar até 2030. Atualmente, no Brasil, os óbitos por hepatite C representam a maior causa de morte entre as hepatites virais (AKAMATSU; SUGAWARA, 2012; BRASIL, 2017).

A hepatite C é causada por um vírus da família Flaviviridae e gênero Hepacivirus. Esse vírus caracteriza-se por ser uma partícula viral esférica, com tamanho entre 40 e $100 \mathrm{~nm}$ de diâmetro; seu material genético é constituído por um RNA de fita simples, polaridade positiva, com aproximadamente 9.400 nucleotídeos, composto por um envoltório e um nucleocapsídeo icosaédrico (CATANESE et al., 2013). Existem, pelo menos, sete genótipos e 67 subtipos do vírus. No Brasil, os genótipos 1 e 3 são os mais prevalentes, sendo que de 50 a $70 \%$ dos casos de hepatite $C$ são causados pelo tipo 17,8,10,11,12,13 (SMITH et al., 2014).

A transmissão do HCV ocorre principalmente por via parenteral, por meio do contato com sangue contaminado, a exemplo do compartilhamento de agulhas, seringas e outros objetos para uso de drogas, reutilização ou falha de esterilização de equipamentos médicos ou odontológicos, falha de esterilização de equipamentos de manicure e reutilização de material para realização de tatuagem, além do uso de sangue e seus derivados contaminados (WHO, 2018). A transmissão sexual do HCV também tem sido relatada de forma esporádica. De modo geral, a transmissão sexual desse vírus é pouco comum e ocorre em relações sem uso de preservativo. Há também a possibilidade de transmissão vertical, em menor proporção dos casos (FAUTEUX-DANIEL et al., 2017). 
O HCV é prevalente em todo o mundo e possui uma ampla distribuição geográfica. Segundo dados do Relatório Global Sobre hepatites cerca de 71 milhões de pessoas no mundo vivem com com infecção crônica pelo HCV, pois, devido a doença ser silenciosa a maioria das pessoas não tem acesso ao diagnóstico e tratamento (BRASIL, 2017).

As hepatites virais estão incluídas na lista de doenças de notificação compulsória do Ministério da Saúde. Os casos de hepatite devem ser registrados no Sistema de Informação de Agravos de Notificação (SINAN) para que sejam geradas informações epidemiológicas com o objetivo de auxiliar no planejamento e na execução das ações de saúde no Brasil e subsidiar a inserção de novas vacinas no Programa Nacional de Imunização. Para tanto, os profissionais de saúde devem realizar os registros dos casos para possibilitar o monitoramento da distribuição da doença e verificar a população predominantemente atingida, além da detecção de surtos e epidemias. O não preenchimento adequado da ficha de notificação pode mascarar a real situação de saúde de uma população, ocasionando intervenções de saúde equivocadas em relação à necessidade local (ANGELOTTI et al., 2013).

Segundo o Ministério da Saúde, de 2007 a 2018, foram detectados no Brasil 210.259 casos de indivíduos que apresentaram anticorpo contra o HCV (anti- HCV)e carga viral (ácido ribonucleico do vírus C - HCV RNA) detectáveis (BRASIL, 2018).

Diante dos preocupantes dados relacionados a hepatite $\mathrm{C}$ nos últimos anos, faz-se necessário um identificar a maior vulnerabilidade para o desenvolvimento desta doença. Dessa forma, este estudo tem como objetivo identificar o perfil epidemiológico de hepatite C no Brasil entre os anos de 2013 a 2018, subsidiando para realizar melhorias no programa de controle da doença para detecção precoce da doença, evitando complicações e, assim promovendo diminuição nos óbitos. 


\section{MATERIAIS E MÉTODO}

Trata-se de um estudo documental, descritivo, de coleta retrospectiva, por meio de casos notificados pelo DATASUS referentes aos agravos de hepatite C diagnosticados no Brasil entre 2013 a 2018.

A coleta de dados foi realizada por meio de tabuladores disponibilizados pelo Ministério da Saúde: Sistema de Informação de Agravos de Notificação (SINANTABNET), seguindo os seguintes passos: 1) acesso ao link http://dtr2004.saude.gov.br/sinanweb/; b) clicar em tabulador de dados; c) selecionar agravo que deseja pesquisar- hepatite; d) acessos aos dados de acordo com as variáveis.

Considerando-se as variáveis como sexo, faixa etária, raça e nível de escolaridade, região do país e fontes de transmissão. As análises ocorrem por meio de técnicas descritivas simples, utilização do programa Microsoft Excel, versão 2016 ferramenta de tratamento de dados e análise de estatísticas simples e construção de tabelas.

Por utilizar o SINAN, uma plataforma do Ministério da Saúde cujos dados estão disponíveis para livre acesso, este estudo não foi submetido à avaliação de um comitê de ética em pesquisa.

\section{RESULTADOS E DISCUSSÃO}

Neste estudo foram confirmados e notificados 264.298 casos de Hepatite no Brasil entre os anos de 2013 a 2018. Para o período estudado, as incidências anuais foram maiores para Hepatite C (128.730 casos confirmados - 48,7\%) e em segundo lugar Hepatite B (96.586 casos confirmados - 36,5\%). Este resultado pode estar relacionado pela transmissão dessa doença pode ocorrer por vias horizontais e verticais, por transfusão sanguínea, uso de drogas injetáveis e terapias invasivas com 
equipamentos contaminados. Atualmente, essa doença é prioridade em políticas públicas de saúde, por serem consideradas um grande problema no Brasil, uma vez que está associada às hepatopatias crônicas e à realização de transplantes de fígado (BRASIL, 2017).

Nesse período no Brasil, foram detectados 128.730 casos de hepatite C (TABELA 1), dos quais 48, 4,1\% (62.329 casos confirmados) ocorreram na Região Sudeste. Historicamente, o Brasil tem apresentado grandes diferenças regionais em seus indicadores epidemiológicos. Esse fato está relacionado, principalmente, o número de habitantes em cada região, às diferentes medidas de acompanhamento e controle de agravos infecciosos executadas nos Estados e municípios. Assim, a compreensão dessas diferenças requer desafios metodológicos, sobretudo quando é considerada a magnitude do território brasileiro em análises de situação de saúde (GONÇALVES et al., 2019).

Ao comparar as taxas de detecção para cada 100 mil habitantes, o Sul se destaca com uma taxa de 24,3 casos, seguida pelo Sudeste com 15,6, Norte 6,3, Centro Oeste 5,9 e por fim Nordeste com 3,2 casos (BRASIL, 2017). Nota-se que em 2017 houve algumas mudanças nos padrões em comparação ao do ano de 2016: Sudeste com $64,1 \%$, Sul com 24,5\%, 5,5 \% no Nordeste, Centro Oeste com 3,3\% e o menor índice permanecendo na Região Norte com 2,5\% (BRASIL, 2018).

Tabela 1. Indicador de Taxa de Incidência de Hepatite C no Brasil, segundo notificação no período de 2013-2018.

\begin{tabular}{l|cc}
\hline Regiões do Brasil & Casos confirmados (n) & Casos confirmados (\%) \\
\hline Região Norte & 6.890 & $5,4 \%$ \\
Região Nordeste & 10.833 & $8,4 \%$ \\
Região Sudeste & 62.329 & $48,4 \%$ \\
Região Sul & 42.886 & $33,3 \%$ \\
Região Centro-Oeste & 5.792 & $4,5 \%$ \\
\hline \multicolumn{1}{c|}{ TOTAL } & $\mathbf{1 2 8 . 7 3 0}$ & $\mathbf{1 0 0}$ \\
\hline
\end{tabular}

Fonte: Ministério da Saúde/SVS- Sistema de Informação de Agravos de NotificaçãoSinan Net, 2020. 
Com relação ao sexo, foi observada maior frequência da infecção por hepatite C (TABELA 2) no gênero masculino (57\%) fato evidenciado por outros estudos (PARABONI, et al, 2012; OLIVEIRA et al,2015). De acordo com Gomes et al (2010) ao analisarem a vulnerabilidade da população para a hepatite $C$, a partir do perfil dos portadores do vírus, constataram que os homens têm mais atitudes que os deixam mais expostos ao vírus como, por exemplo, uso de drogas injetáveis ou inaláveis, consumo de álcool e a prática de relações sexuais sem uso de preservativo.

Quanto à faixa etária, a maioria dos pacientes acometidos com essa infecção possuem idade entre 40 e 59 anos (68.746 casos confirmados - 53,4\%) (TABELA 2 ) corroboram com estudos de Mello et al (2011), Oliveira et al (2015) e Oliveira et al (2018). No Brasil, a média de idade casos de hepatite $C$, para ambos os sexos, é de 46,3 anos (BRASIL, 2018). A hepatite C é uma infecção latente e muitas vezes silenciosa, com apresentação do quadro clínico tardio e, provavelmente por isso, a maior prevalência dos casos esteja em indivíduos com idade superior a 50 anos, sugerindo infecção em passado distante (CATANESE et al., 2013; OLIVEIRA et al.,2018).

Importante ressaltar que como dito anteriormente, a hepatite $C$ é uma infecção silenciosa e latente, com manifestações de quadro clínicos tardios e por conta dessa forte característica a maior prevalência esteja em indivíduos com a idade acima de 50 anos, indicando que a infecção foi adquirida em um passado distante, onde não haviam métodos básicos de biossegurança, como a correta esterilização de agulhas e seringas em procedimentos dentários, cirúrgicos e até mesmo na realização de tatuagens, perfuração com piercing e procedimentos em salões de beleza com o uso de alicate de unhas por manicures que não realizavam a correta esterilização dos materiais, sendo assim um grande meio de contágio para o HCV, tendo em vista a grande rotatividade do local. Outro fator determinante foi a ausência de testes de triagens para detecção do HCV em bolsas de sangue e hemoderivados nos hemocentros do País antes de novembro de 1993, onde a detecção do anti-HCV não era obrigatória, infectando assim, hemofílicos e usuários de bolsa de sangue casualmente, como em caso de acidentes (OLIVEIRA et al., 2018). 
Em relação à raça, observa-se que maioria é branca com 53,6\% (TABELA 2) dos casos notificados. Esse aspecto não se distribui uniformemente nos estudos na literatura nas unidades da federação devido heterogeneidade da miscigenação no país.

Tabela 2. Casos de Hepatite $C$ segundo as características sóciodemográficas no período de 2013-2018 no Brasil.

\begin{tabular}{l|cc}
\hline \multicolumn{1}{c}{ Variáveis } & Casos confirmados (n) & Casos confirmados (\%) \\
\hline Sexo & & \\
\hline Feminino & 55.208 & $42,9 \%$ \\
Masculino & 73.458 & $57 \%$ \\
Ignorado & 64 & $0,1 \%$ \\
\hline Escolaridade & & \\
\hline Analfabeto & 1.795 & $1,4 \%$ \\
4 Série Incompleta & 6.754 & $5,2 \%$ \\
4 Série Completa & 9.788 & $7,6 \%$ \\
Ens. Fund. Incompleto & 17.769 & $13,8 \%$ \\
Ens. Fund. Completo & 10.992 & $8,5 \%$ \\
Ens. Médio Incompleto & 6.776 & $5,2 \%$ \\
Ens. Médio Completo & 21.690 & $16,8 \%$ \\
Ed. Superior Incompleto & 2.917 & $2,2 \%$ \\
Ed. Superior Completo & 7.822 & $6 \%$ \\
Ignorado & 42.143 & $32,7 \%$ \\
Não se aplica & 884 & $0,6 \%$ \\
\hline
\end{tabular}

\section{Raça}

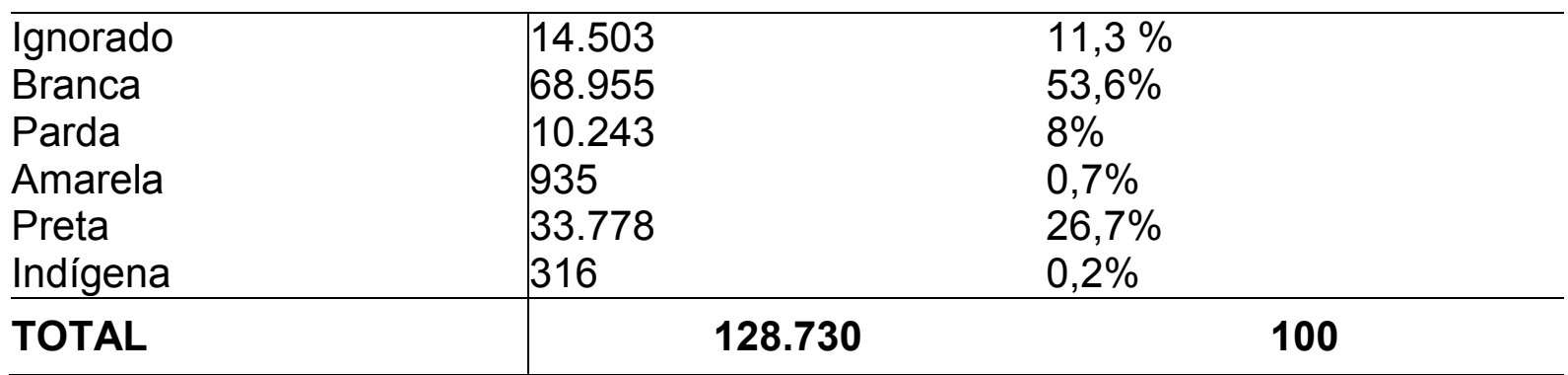

Fonte: Ministério da Saúde/SVS- Sistema de Informação de Agravos de NotificaçãoSinan Net, 2020.

Quanto ao grau de escolaridade, predominou-se ao ignorado (32.737 casos notificados - 32,7\%) (TABELA 2) nesse período de estudo. Em segundo lugar com $16,8 \%$ notificações se encontra o grupo de pessoas com ensino médio completo. $O$ 
restante dos casos corresponde em maior parte às pessoas com no máximo ensino Fundamental incompleto. Diante disso, é perceptível que não se pode traçar um perfil adequado dos casos de hepatite $\mathrm{C}$ no período em questão em termos de escolaridade, pois mais da metade dos casos foi negligenciada em termos de notificação adequada. Afirma-se que um dos motivos para isso pode ser a quantidade de questões e páginas a preencher, o que contribui para o preenchimento inconsistente ou o não preenchimento (CORDEIRO, OLIVEIRA JÚNIOR, 2018). Dessa forma, ressalta-se a importância do preenchimento correto e da completude dos campos da ficha de notificação no momento da investigação epidemiológica, uma vez que a vigilância epidemiológica é uma ferramenta imprescindível para o entendimento da dinâmica de epidemias por HCV.

Tabela 3. Casos de Hepatite C de acordo fonte de transmissão no período de 20132018 no Brasil.

\begin{tabular}{l|cc}
\hline \multicolumn{1}{c|}{$\begin{array}{c}\text { Fontes de transmissão } \\
\text { Hepatite C }\end{array}$} & Casos confirmados (n) & Casos confirmados (\%) \\
\hline Ignorado & 73.703 & 57,3 \\
Sexual & 12.482 & 9,7 \\
Transfusional & 12.028 & 9,3 \\
Uso de drogas Injetáveis & 14.189 & 11 \\
Vertical & 322 & 0,3 \\
Acidente de trabalho & 504 & 0,4 \\
Hemodiálise & 834 & 0,6 \\
Domiciliar & 625 & 0,5 \\
Tratamento cirúrgico & 4.525 & 3,5 \\
Tratamento dentário & 2.857 & 2,2 \\
Pessoa/pessoa & 1.463 & 1,1 \\
Alimentos/água & 74 & 0,06 \\
Outros & 5.114 & 4 \\
\hline TOTAL & $\mathbf{1 2 8 . 7 3 0}$ & $\mathbf{1 0 0}$ \\
\hline
\end{tabular}

Fonte: Ministério da Saúde/SVS- Sistema de Informação de Agravos de NotificaçãoSinan Net, 2020.

Quanto à provável fonte ou mecanismo de transmissão dos casos notificados para a hepatite $\mathrm{C}$ (TABELA 3), dados relatados nesse período de estudo demonstram que em mais da metade $(57,3 \%)$ dos casos essa informação foi registrada como "ignorada”, dificultando uma melhor avaliação sobre as prováveis fontes de infecção, 
semelhante aos dados obtidos no estudo (RODRIGUES et al., 2019). Acredita-se que este fato possa estar relacionado à dificuldade que se tem de estabelecer um fator único como o responsável pelo desenvolvimento da infecção (BRASIL, 2018). Em outros estudos realizados sobre hepatites C sobressai a transmissão por via sexual, transfusional e o uso de drogas injetáveis, dados que corroboram os resultados desta pesquisa, que apresentou, além desses mecanismos de transmissão, a via cirúrgica e a tratamento dentário (SANTOS; MORAIS, 2018).

Além disso, estudos demostram que o uso de drogas pode ser considerado com um dos mais importantes fatores de risco de transmissão do HCV, sendo responsável por novos casos todos os anos no Brasil (TABELA 3). As altas taxas de prevalência são atribuídas ao uso de seringas contaminadas e o compartilhamento de agulhas entre diversos usuários. Outros objetos utilizados para o uso de drogas como canudos para a aspiração da cocaína ou cachimbos para uso de crack, podem causar lesões nas mucosas nasais e labiais, e desse modo, mesmo que uma quantidade mínima de sangue, pode conter uma carga viral suficiente para desencadear uma infecção. Por conta disso, pesquisas apontam que regiões metropolitanas com altos índices populacionais estejam mais propensas à alta incidência de HCV, por conta da marginalização dos indivíduos, que são mais vulneráveis ao uso de drogas (RODRIGUES, 2012).

Porém, no ano de 2017 a principal fonte de infecção foi a por via sexual, $(9,2 \%)$ seguida pelo uso de drogas $(8,1 \%)$ e por último a por via transfusional $(6,8 \%)$, de (BRASIL,2018). Em relação ao risco de contágio do HCV por via sexual, nota-se um aumento gradativo dessa via ao longo dos anos. Esse modo de transmissão ainda não está completamente elucidado, e vem sendo fonte de contínuos estudos. A maior prevalência observada na literatura está em indivíduos que são atendidos em unidades hospitalares ou clínicas especificas para doenças sexualmente transmissíveis, por conta da maioria desses pacientes apresentarem coinfecção com outros vírus como HIV e HBV.

A hepatite C representa impacto significativo para a saúde pública em decorrência da progressão da doença e de suas complicações, pois não apresenta vacina, a evolução silenciosa, diagnóstico tardio e, nas fases avançadas, o paciente pode evoluir para óbito. Estima-se que, no mundo, a hepatite $\mathrm{C}$ seja responsável por 
aproximadamente 700.000 óbitos anuais (WHO, 2018). No Brasil, os números de óbitos causados pelo HCV são superiores em comparação com os óbitos das demais hepatites virais. Esses números vêm aumentando a cada ano em todas as Regiões do país. No período de 2000 a 2016 foram notificados 50.179 óbitos relacionado à hepatite C no Brasil, enquanto no período de 2012 a 2017 foram notificados 11.903 óbitos dessa patologia. No ano de 2016 as regiões Sul e Sudeste apresentaram coeficiente de mortalidade excedente à média nacional $(1,0)$, com 1,7 e 1,2 por 100 mil habitantes respectivamente (BRASIL, 2017).

Outro ponto importante, é o número de casos que são descobertos somente na fase crônica da doença. Os índices de rastreamento da hepatite $C$ na fase aguda são baixos e pouco notificados, esse fato provavelmente se por conta da sensibilidade e especificidade dos testes diagnóstico e ausência de exames imunológicos mais precisos. Esse conjunto de fatores, a hepatite $\mathrm{C}$ é a mais letal dentre todas as outras hepatites, com altos índices de quadros clínicos de hepatocarcinoma e cirrose, que em sua maioria evoluem para um transplante ou óbito.

\section{CONCLUSÕES}

As informações apresentadas demonstram a hepatite $C$ é um grave problema de saúde no país. Os dados compilados sinalizam para o perfil dos brasileiros atingidos corresponde em sua maioria homens, raça branca e com idade entre 40 a 59 anos. No entanto, a escolaridade e fonte de transmissão foram notificados na maior parte dos casos como "ignorado" e que, números de óbitos causados pelo HCV são superiores em comparação com os óbitos das demais hepatites virais.

Dessa forma, ressalta-se a importância do preenchimento correto da ficha de notificação para definir o verdadeiro panorama da Hepatite C no Brasil e assim lançar melhores estratégias de planejamento e/ou incremento de ações específicas para promoção de saúde e prevenção, com campanhas de alertas e orientações, além de atividades frequentes de educação em saúde considerando grupos/situações de 
risco, para diminuir incidência no país e, assim, contribuir para melhorar a qualidade de vida.

\section{REFERÊNCIAS BIBLIOGRÁFICAS}

AKAMATSU, N.; SUGAWARA, Y. Liver transplantation and hepatitis C. International Journal of Hepatology, New York, v. 2012, p. 686135, 2012.

ANGELOTTI LCZ, ALEXANDRE PBD, MIRANZI SSC, SCATENA LM. Qualidade de dados de notificação e acompanhamento dos casos de tuberculose em Minas Gerais. Revista Enfermagem Atenção Saúde, v.2, n.2, p.84-98, 2013.

BRASIL. Ministério da Saúde. Secretaria de Vigilância em Saúde. Departamento de Vigilância, Prevenção e Controle das IST, do HIVIAids e das Hepatites Virais. Boletim epidemiológico. Brasília: Ministério da Saúde; 2017.

BRASIL. Ministerio da Saude. Secretaria de Vigilancia em Saude. Departamento de Vigilancia, Prevencao e Controle das IST, do HIVIAids e das Hepatites Virais. Protocolo Clínico e Diretrizes Terapêuticas para Prevenção da Transmissão Vertical do HIV, Sífilis e Hepatites Virais. Brasilia: Ministerio da Saude, 2018.

CATANESE MT, URYU K, KOPP M, EDWARDS TJ, ANDRUS L, RICE WJ, et al. Ultrastructural analysis of hepatitis $C$ virus particles. Proceedings of the National Academy of Sciences, v.110 n. 23, 2013.

CORDEIRO TMSC, D'OLIVEIRA JÚNIOR A. Qualidade dos dados das notificações de hepatites virais por acidentes de trabalho, Brasil. Revista Brasileira Epidemiologia v, 21, p.1$11,2018$.

FAUTEUX-DANIEL, S. et al. Vertical Transmission of Hepatitis C Virus: VariableTransmission Bottleneck and Evidence of Midgestation In Utero Infection. Journal of Virology, [S.I.], v. 91, n. 23, dez. 2017.

GOMES DT, TOCANTINS FR, SOUZA FBA. Perfil de portadores de hepatite C e a vulnerabilidade da população: potencialidades para a enfermagem. Revista Pesquisa Cuidado Fundamental (Online), out-dez; 2 (supl): 512-5., 2010.

GONÇALVES, NELSON VEIGA; MIRANDA, CLAUDIA DO SOCORRO CARVALHO; GUEDES, JUAN ANDRADE; SILVA, LARISSA DE CÁSSIA TORK DA; BARROS, ELIZABETH MACIEL; TAVARES, CILEIDE GOMES DA MOTA; PALÁCIOS, VERA REGINA DA CUNHA MENEZES; COSTA, SIMONE BEVERLY NASCIMENTO DA; OLIVEIRA, HELENA CUNHA; XAVIER, MARÍLIA BRASIL. Hepatites $B$ e $C$ nasáreas de três Centros Regionais de Saúde do Estado do Pará, Brasil: uma análise espacial, epidemiológica e socioeconômica. Caderno saúde coletiva, v.27, n.1 p.1-10, jan.- mar. 2019.

MELLO JC, MOTTA TP, SANTOS MC. Perfil epidemiológico de portadores de hepatite C do Núcleo Hospitalar Epidemiológico do sul do Brasil. Ensaio Ciencia, v.15, n.3, p.55-64, 2011.

OLIVEIRA JM, MAIA MAC, SILVA JP, QUEIROZ CA, FORTUNA CM, ANDRADE RD. Perfil epidemiológico de portadores de hepatite $C$ : estudo descritivo em unidade de referência regional. Revista Pesquisa Cuidado Fundamental (Online). v.7, n.4, p.3454-66, out-dez 2015. 
OLIVEIRA, T. J. B., LIDYANNE, A. P., DOS REIS, L. A. P., DOS, BARRETO, L. S. L. O., GOMES, J. G., MANRIQUE, E. J. C. Perfil epidemiológico dos casos de hepatite $C$ em um hospital de referência em doenças infectocontagiosas no estado de Goiás, Revista PanAmazônica de Saúde, v.9, n.1 , 2018.

PARABONI MLR, SBEGHEN MD, WOLFF FH, MOREIRA LB. Risk factors for infection with different hepatitis $C$ virus genotypes in southern Brazil. Science World Journal. 2012.

RODRIGUES N. et al. Prevalência da hepatite viral C em adultos usuários de serviço público de saúde do município de São José dos Pinhais - Paraná. Revista Brasileira de Epidemiologia, São Paulo, v.15, n.3, p.627 - 638, Set 2012.

RODRIGUES LMC, FURTADO EZL, OLIVEIRA AKN, MORAIS JC, RESENDE MTS, SILVA VR. Mapeamento epidemiológico das hepatites hospitalares. Revista Brasileira Promoção de Saúde, v. 32, p.87-14, 2019.

SANTOS ACS, MORAIS MTM. Perfil epidemiológico e sociodemográfico dos portadores de hepatite B de um município do Sudoeste Baiano. Revista Saúde v.14, n.1, p.1073-80, 2018.

SMITH, D. B. et al. Expanded Classification of Hepatitis C Virus Into 7 Genotypes and 67 Subtypes: Updated Criteria and Genotype Assignment Web Resource. Hepatology, [S.I.], v. 59, n. 1, p. 318-327, jan. 2014.

WHO (WORLD HEALTH ORGANIZATION). Hepatitis c [On-line]. Disponível em: <https://www.who.int/news-room/fact sheets/detail/hepatitis-c>. Acesso em: 24 fev. 2019. 